\title{
A choice between transcriptional enhancement and repression by the v-erbA oncoprotein governed by one nucleotide in a thyroid hormone responsive half site
}

\author{
ML Andersson ${ }^{1}$ and B Vennström*,1 \\ ${ }^{1}$ Department of Cell and Molecular Biology (CMB), Karolinska Institute, Box 285, S-171 77 Stockholm, Sweden
}

\begin{abstract}
The v-erbA oncoprotein (P75 $\left.{ }^{\text {gag-v-erbA }}\right)$ can repress thyroid hormone receptor induced transcriptional activation of target genes. A central question is how hormone responsive elements in a target gene determine the transcriptional regulation mediated by $P 75^{\text {gag-v-erbs }}$. We addressed this with receptors chimeric between P75 ${ }^{\text {ag-v-erbA }}$ and thyroid hormone receptor (TR) by testing their regulatory activities on thyroid hormone response elements (TREs) differing in the sequence of the consensus core recognition motif AGGTCA. We report here that enhances, TR dependent transcriptional activation is conferred by $P 7^{\text {gag-v-erbA }}$ when the thymidine in the half site recognition motif is exchanged for an adenosine. The enhancement was independent of the DNA binding region of $\mathrm{P}^{\text {gag-v-erbs }}$, whereas increased expression of corepressor abolished the enhancing effect. The data indicate that the enhancement results from an impaired DNA binding by the oncoprotein combined with an effective scavenging of corepressors. Our data thus suggest the $P 7^{\text {gag-v-erbs }}$ indirectly can contribute to enhancement of thyroid hormone induced gene expression. Oncogene (2000) 19, 3563-3569.
\end{abstract}

Keywords: thyroid hormone; receptor; P75 $5^{\text {gag-v-erbA; }}$ corepressor; response element

\section{Introduction}

P75 ${ }^{\text {gag-v-erbA }}$, the oncoprotein homologue of the thyroid hormone receptor $\alpha(\mathrm{TR} \alpha)$, contains several alterations: an N-terminal fusion to retroviral gag sequences, 13 interspersed amino acid substitutions, and two small deletions in the $\mathrm{N}$ - and $\mathrm{C}$-termini, respectively (Sap et al., 1986; Weinberger et al., 1986). The amino acid substitutions in $\mathrm{P} 75^{\text {gag-v-erba }}$ has led to a dysfunction in transcriptional activation due to the inability to bind thyroid hormone (T3) (Munoz et al., 1988; Privalsky et al., 1988). Consequently, P75 $5^{\text {gag-v-erbA }}$ does not undergo the conformational changes induced by binding of ligand (Andersson et al., 1992; Yen et al., 1992) and is thus impaired in recruiting coactivators (Chen and Evans, 1995). In absence of ligand however, both TR and $\mathrm{P} 75^{\text {gag-v-erbA }}$ function as transcriptional repressors. The substitutions in the ligand binding domain (LBD) of P75 gag-verbA that abolish the binding of T3 have been suggested to be required for the ability of P75 gag-v-erbA to cause erytholeukemia (Schroeder et al., 1992a,b). The

*Correspondence: B Vennström

Received 4 November 1999; revised 17 May 2000; accepted 18 May 2000 paradigm view of $\mathrm{P} 75^{\text {gag-v-erbA }}$ in transcription is that P75 ${ }^{\text {gag-v-erbA }}$ functions as a dominant transcriptional repressor on activated transcription induced by TR (Damm et al., 1989; Disela et al., 1991; Zenke et al., 1988).

Hormone response elements for TR (TREs) have been identified in upstream or intron regions of several genes that are important in development and in homeostasis (Baniahmad et al., 1990; Desai-Yajnik et al., 1995; Farsetti et al., 1992; Glass et al., 1987; Petty et al., 1990; Tsika et al., 1990; Wahlström et al., 1992; Wong et al., 1997). These elements contain AGGTCA or AGGACA hexamers in which two or more motifs are positioned as direct repeats (DR), or as everted repeats (ER) separated by 4-6 nucleotide (Näär et al., 1991; Umesono et al., 1991; Wahlström et al., 1992). P75 ${ }^{\text {gag-v-erbA }}$ binds in vitro to oligonucleotides containing configurations of either the DR or the ER type (Andersson et al., 1992; Wahlström et al., 1992) although it exhibits a higher affinity for the latter element (Wahlström et al., 1996). The normal thyroid hormone receptor, in contrast to P75 $5^{\text {gag-v-erbA }}$, binds efficiently to half sites containing the sequence AGGACA (Judelson and Privalsky, 1996; Nelson et al., 1994).

Transcription factors are dependent on cofactors in their regulation of activation or repression. Specific repressor proteins, denoted corepressors, were proposed in 1994 (Casanova et al., 1994; Rascle et al., 1994) and characterized in 1995 (Chen and Evans, 1995; Horlein et al., 1995; Kurokawa et al., 1995). The two corepressors N-Cor and SMRT, recruited by several nuclear receptors, are ubiquitous in mammalian cells and they form repressor complexes in association with deacetylases (HDAC) (Chen et al., 1996). The TR (or P75 ${ }^{\text {gag-v-erbA }}$ ) interaction with SMRT occurs through the hinge domain located in the N-terminal portion of LBD (Lee and Mahdavi, 1993; Nawaz et al., 1995; Zamir et al., 1997). It has also been suggested that the extreme C-terminus of the LBD is involved in binding corepressors (Baniahmad et al., 1998; Li et al., 1997; Tagami et al., 1998).

In this study we sought to understand how P75 ${ }^{\text {gag-v-erbA }}$ interferes with thyroid hormone receptor activated transcription. We used the response element F2 from the chicken lysozyme gene which contained AGGTCA or mutant AGGACA half sites in an everted configuration, and tested the ability of P75 ${ }^{\mathrm{gag}-}$ verbA to interfere with gene regulation by TR. We show here that the fourth nucleotide in the half site of the treatment determines whether P75 ${ }^{\text {gag-v-erbA }}$ mediates repression or enhanced activation of TR activated transcription. 


\section{Results}

\section{Repression specificity by P75 gag-v-erbA}

The N-terminal and DNA binding domains (DBD) or TR and P75 gag-verbA, that differ in four amino acid residues, are important for proper recognition of central nucleotides in the TRE half sites (Nelson et al., 1994; Smit-McBride and Privalsky, 1994). To investigate the influence of these changes on the repression properties of $\mathrm{P} 75^{\text {gag-v-erbA }}$ with different $\mathrm{TRE}$ half site sequences, we constructed plasmids encoding chimeric proteins containing the LBD from P75 gag-v-erbA and various portions of the DBD and N-terminal regions from TR (Figure 1b). These constructs were tested for repression of $\mathrm{T} 3$ inducible gene expression through two different TREs (Figure 1a). The first TRE, derived from the chicken lysozyme regulatory region (Baniahmad et al., 1990), was synthesized as two tandem copies of the everted repeat and cloned into a luciferase reporter plasmid (F2Tx2). The second TRE differs by containing an adenosine instead of a thymidine in the fourth position of the half site (AGGACA in F2Ax2, Figure 1b). We first compared the repression activities of $\mathrm{P} 75^{\text {gag-v-erbA }}$ and a chimera, c56, that contained two TR-specific amino acid replacements in the ligand binding domain, since all other chimeras were based on the c56 construct.

Equimolar or threefold excess of P75 $5^{\text {gag-v-erbA }}$ or c56 expressing plasmids were thus transfected into cells along with a TR expressing plasmid. Since some of our expression plasmids are based on retrovirus vector constructs with LTRs maximally active in avian cells, quail QT6 cells were chosen to conduct the protein expression. Extracts from cells, transfected in triplicates and grown in absence or presence of thyroid hormone, were analysed for luciferase activity. The results show that P75 ${ }^{\text {gag-v-erbA }}$ and c56 repressed to the same extent both the basal and T3 induced levels of transactivation through the F2Tx2 element (Figure 2a). However, both proteins failed to repress TR activation on the F2Ax2 element, and mediated, surprisingly, a 1.5-2-fold enhancement of TR activation at different plasmid ratios (Figure 2a). Our data show that the repression by $\mathrm{P} 75^{\text {gag-v-erbA }}$ on TR activation was highly dependent on the nucleotides in the half site of the response element, and that the c56 protein has properties very similar to those of the viral protein.

\section{DNA binding capacities}

The transfection results prompted us to test if the abolished repressor function of $\mathrm{P} 75^{\text {gag-v-erbA }}$ on the F2Ax2 element was a result of low DNA binding affinity. We therefore tested the binding of TR and of P75 gag-v-erbA to the two response elements in gel mobility assays. TR or P75 ${ }^{\text {gag-v-erbA }}$ extracts produced in HeLa infected with recombinant Vaccinia virus were mixed with ${ }^{32} \mathrm{P}$-labeled oligonucleotides containing the F2Tx2 or the F2Ax2 sequences, and the mobility of complexes was analysed. Previous studies have shown that $\mathrm{P} 75^{\text {gag-v-erbA }}$ and $\mathrm{TR}$ bind in vitro to everted repeated elements as homodimers with high affinity (Andersson et al., 1992), and we therefore tested their binding to the F2T and F2A element by adding increasing amounts of the oligonucleotide containing the AGGT-
A

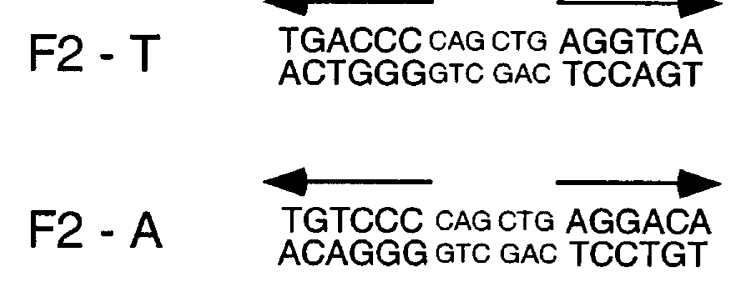

B

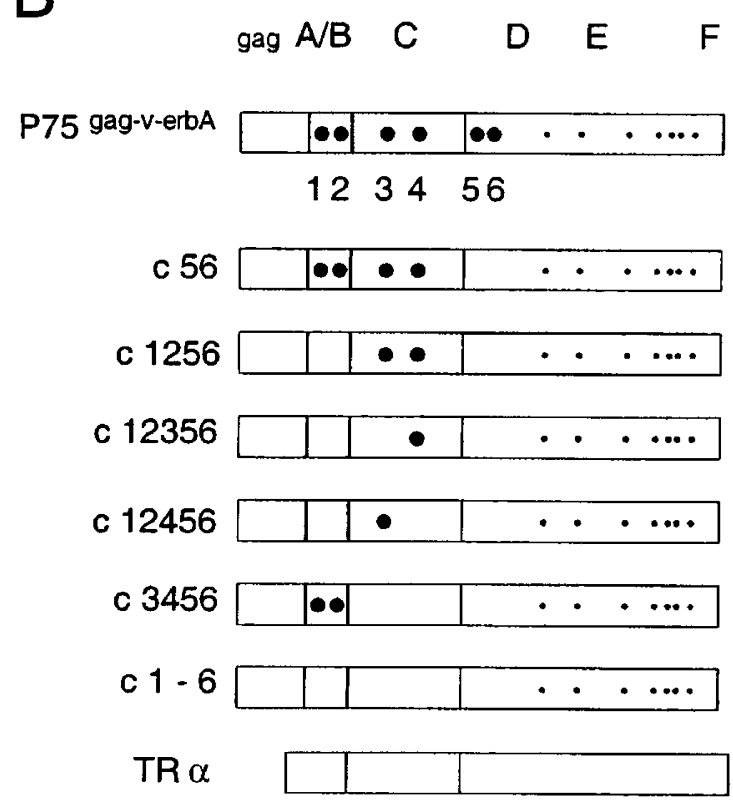

v-erbA - LBD

Figure 1 Overview of the $\mathrm{P} 75^{\text {gag-v-erbA }} / \mathrm{TR}$ proteins and response elements used. (a) The F2-T response element sequence is identical to the chicken lysozyme upstream control region F2. The F2-A element is identical to the F2-T element except for the adenosine in place of the thymidine at the fourth nucleotide of the half site. Both elements were synthesized and used as a tandem repeat (F2Tx2 and F2Ax2). (b) Alignment of the P75 $5^{\text {gag-v-erbA }}, T R$ and variant proteins that are chimeric between TR and the oncoprotein. The different individual amino acids between P75 $5^{\text {gag-v-erbA }}$ and TR are indicated by dots. The amino acids addressed in this paper are numbers 1-6: Amino acid 1, His (Arg in TR); amino acid 2, Cys (Tyr in TR); amino acid 3, Ser (Gly in TR); amino acid 4, Thr (Lys in TR); amino acid 5, Arg (Lys in TR); amino acid 6, Leu (Pro in TR). The nomenclature for nuclear hormone receptor domains is: A/B, N-terminal; C, DBD; $\mathrm{D}$, hinge domain; E, LBD; F, AF-2; shown at the top. The $\mathrm{P} 75^{\text {gag-v-erbA }}$ is a fusion protein with sequences derived from the avian erythroblastosis virus (gag). A truncated v-erbA protein containing the LBD and lacking the entire N-terminal and the DBD (v-erbA-LBD) was also used

CA half site. The results show first that TR binds more efficiently than P75 gag-v-erbA to either of the elements. Secondly, binding of P75 $5^{\text {gag-verbA }}$ to the AGGACA element was easily competed out by the AGGTCA oligonucleotide, as compared to what was seen with the AGGTCA element (Figure 2b). The data show that the fourth nucleotide in the half site sequence is critical for high affinity DNA binding by $\mathrm{P} 75^{\text {gag-v-erbA }}$. 

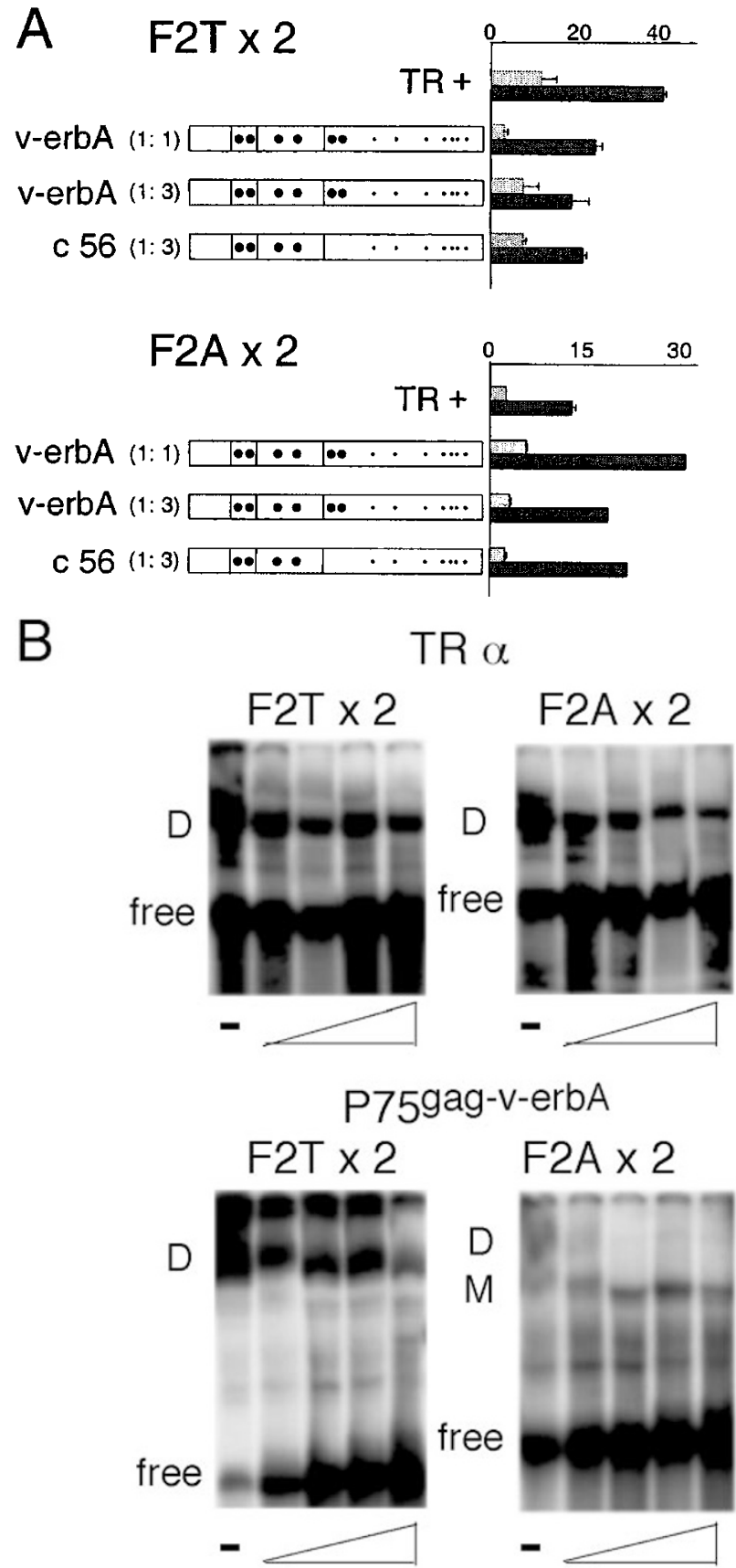

Figure 2 Enhanced activation or repression of TR activated transcription depend on sequences in the response element. (a) A TR expressing plasmid was cotransfected into QT6 cells along with reporter plasmids containing the F2Tx2 (upper panel) or the F2Ax2 (lower panel) elements in absence (TR + ) or in presence of P75 $5^{\text {gag-v-erbA }}$ (v-erbA), or of c56 expressing plasmid at the indicated excess ratios. Cells were grown in absence (light gray) or in presence (dark gray) of $\mathrm{T} 3$. The $\mathrm{X}$-axis shows luciferase activity in arbitrary units. (b) In vitro DNA binding by TR (upper panels) and by P75 gag-v-erbA (lower panels) as detected by gel mobility assays. Extracts containing Vaccinia virus expressed TR or v-erbA were mixed with ${ }^{32} \mathrm{P}$-labeled F2Tx2 or F2Ax2 oligonucleotide in absence $(-)$ or in presence of increased amounts $(1.4,2.9,4.8$ or 14 pmoles $)$ of the F2Tx2 oligonucleotide and analysed in an acrylamide gel. D, receptor dimers; $\mathrm{M}$, monomers; free, excess oligonucleotides not bound to proteins

\section{Identification of amino acid residues in P75 gag-verbA important for enhanced transactivation}

The low binding affinity of P75 $5^{\text {gag-v-erbA }}$ to the F2Ax2 TRE element in vitro raised the possibility that the mutations in the N-terminus or in the DBD of P75 ${ }^{\text {gag-v-erbA }}$ were responsible for the enhancement of TR activated transcription, a hypothesis supported by independent evidence: substitution of glycine 61 in the wild type TR for a serine (Gly61Ser) decreases DNA binding to elements containing an AGGACA half site, and reduces T3-dependent transactivation (Chen et al., 1993; Nelson et al., 1994). The amino acid residues specific to $\mathrm{P} 75^{\text {gag-verbA }}$ were therefore changed to the corresponding residues of TR: histidine 12 to arginine (His12Arg; amino acid change 1 in Figure 1b) located in a region of basic amino acids that in TR encompasses part of AF-1 (Hadzik et al., 1995); Cys32Tyr (amino acid 2) locate N-terminal of the DBD; Ser61Gly (amino acid 3) located in the DBD recognition sequence important for specific binding to DNA (P-box); and Thr78Lys (amino acid 4) located in the D-box, potentially mediating dimerization with partner molecules. Combinations of these replacements were introduced into $\mathrm{P} 75^{\mathrm{gag}-\mathrm{v}-\mathrm{erbA}}$, and the resulting constructs tested for repression and enhancement of TR induced activation via the AGGTCA and AGGACA elements. First, the chimeric protein $\mathrm{c} 1-6$, containing the first six TR-specific amino acid residues in the N-terminus and the DBD, was compared to the control protein c56. These two chimeras differed in their transcriptional properties on the F2Ax2 element: c1-6 failed to mediate the enhancement of TR dependent activation seen with c56 on the F2Ax2 element, and instead it caused a twofold repression Figure 3a). This shows that the four first amino acid replacements of TR, when inserted in the N-terminus and in the DBD of P75 ${ }^{\text {gag-v-erbA }}$, do not enhance but repress the TR activated transcription on the $\mathrm{F} 2 \mathrm{Ax} 2$ response element.

These results raised the question, which of the four TR-specific amino acid residues was the most important for TR dependent enhancement of transcription via the F2A element. For this, the chimeras c1256 and c12456 (Figure 1b) were tested for repression and enhancement of TR activation as described above. The results show that expression from the two constructs poorly repressed activation via the AGGTCA element, and that the enhancement on the AGGACA element was as efficient as observed with c56 (Figure 3a). To analyse the importance of the Ser16Gly replacement, located in the P-box, we tested the c12356 construct. This chimeric protein repressed as efficiently as the control c56 on the AGGTCA element, whereas it failed to either repress or enhance on the AGGACA TRE (Figure 3a).

Finally, we tested the c3456 construct, which encodes a protein containing the DBD from TR and the $\mathrm{N}$ terminus of P75 ${ }^{\text {gag-v-erba }}$. On the F2Tx2 element and in presence of hormone, this chimera was more efficient in repression than the control c56 protein whereas on the F2Ax2 element it neither enhanced nor repressed TR induced transcription (Figure 3a). It is noteworthy that the two N-terminal amino acids of P75 ${ }^{\text {gag-v-erbA }}$ confer an increased repression in absence of hormone as compared to the TR-specific residues (Figure 3a). We conclude that serine 61, located in the P-box of the DNA binding domain of P75 gag-v-erbA is a major determinant for allowing enhancement of $\mathrm{T} 3$ induced transcription via the F2A element. 
A

F2T $\times 2$

F2A $\times 2$
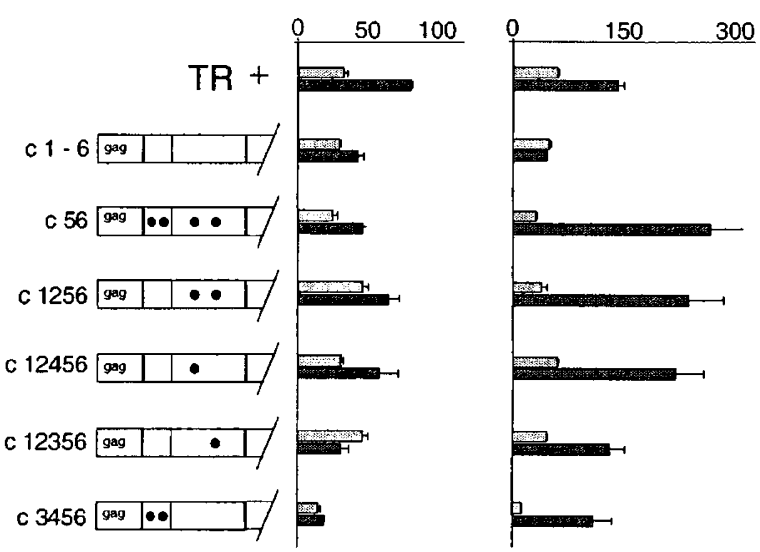

B

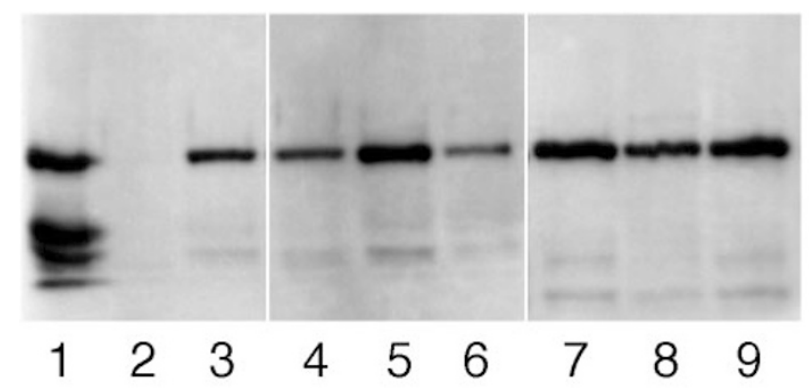

Figure 3 Transcriptional effects of P75 $5^{\text {gag-v-erbA }}$ variant proteins. (a) Repression or enhancement of TR activated transcription by chimeric proteins was assayed by contransfecting TR $\alpha$ expressing plasmids along with vectors that encode P75 $75^{\text {gag-v-erbA }}$ or TRP75 $5^{\text {gag-v-erbA }}$ chimeras as indicated. activity is shown in absence (light gray) and in presence of hormone (dark gray). Luciferase activity is shown as arbitrary units. (b) Western blot analysis of $\mathrm{TR} /$ gag-v-erbA proteins. Lane 1, Vaccinia produced P75 ${ }^{\text {gag-v-erbA; }}$ lane 2, Vaccinia control extract; lane 3, c1256; lane 4, c12456; lane 5, 12356; lane 6, c3456; lane 7, c56; lane 8, c1-6; lane 9, retrovirus produced $\mathrm{P} 75^{\text {gag-v-erbA }}$

To verify that $\mathrm{P} 75^{\text {gag-v-erbA }}$ and the chimeric proteins were expressed with similar efficiencies from the constructs, nuclear extracts were prepared from cells infected with retrovirus vectors expressing the P75 $5^{\text {gag-v-erbA }}, \mathrm{c} 56, \mathrm{cl}-6, \mathrm{c} 1256, \mathrm{c} 12456, \mathrm{c} 12356$ and c3456 proteins respectively. Figure $3 b$, representing a Western blot developed with anti-TR antibodies, shows that the proteins were expressed at similar levels, and that they had the same size as the control P75 ${ }^{\text {gag-v-erbA }}$

\section{Role of the DBD}

The observation that some of the chimeric proteins enhanced rather than repressed TR induced transcription raised the question if the ligand binding domain of P75 gag-v-erbA, without the DBD, also would enhance TR induced transcription. Accordingly, the P75 ${ }^{\text {gag-v-erbA }}$ LBD was tested for effects on TR activation as described above. The results show that the LBD plasmid in a threefold excess failed to repress, and instead conferred a threefold
A

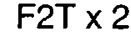

$\mathrm{F} 2 \mathrm{~A} \times 2$

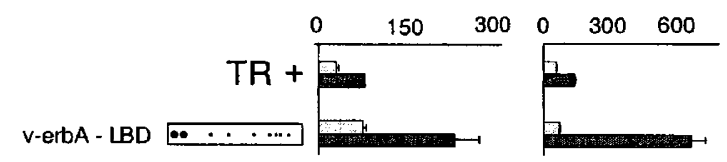

B

$\mathrm{F} 2 \mathrm{~A} \times 2$

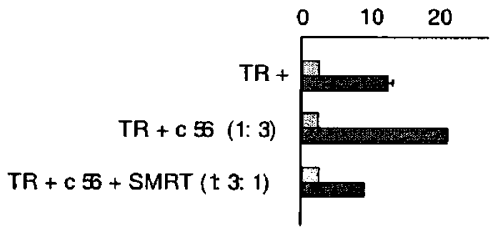

C

$T R+T 3$

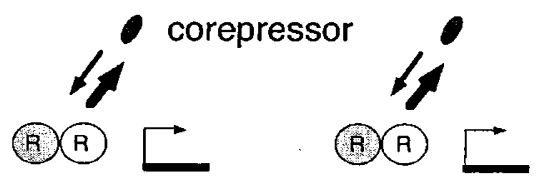

$\mathrm{TR}+\mathrm{T3}$

(B)

+ v-erbA

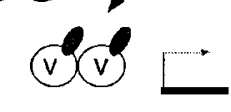

(B) $(\mathrm{R})$

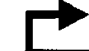

AGGACA

Figure 4 Transcriptional effects by the ligand binding domain of P75 $5^{\text {gag-v-erbA }}$ and by corepressors. (a) A plasmid encoding TR $(\mathrm{TR}+)$ together with a plasmid encoding the LBD of P75 $5^{\text {gag-v-erbA }}$ (v-erbA-LBD) were cotransfected in a ratio of $1: 3$ together with the F2Tx2 or the F2Ax2 reporter constructs. (b) A TR plasmid $(\mathrm{TR}+)$ was cotransfected with the c56 expressing plasmid and the $\mathrm{F} 2 \mathrm{Ax} 2$ reporter plasmid in absence $(\mathrm{TR}+\mathrm{c} 56)$ or presence of corepressor (TR + c56+SMRT) at indicated ratios. Light gray, in absence of T3; dark gray, in presence of T3. (c) A possible model for enhanced activation by P75 $5^{\text {gag-v-erbA }}$. Half sites containing a thymidine or an adenosine in the fourth position mediate TR activated transcription ( $\mathrm{TR}+\mathrm{T} 3)$. Corepressors bind dynamically to TR, and their release is favored by T3 binding (upper panels). The P75 gag-v-erbA molecules compete with $\mathrm{TR}$ for binding to response element that contain AGGTCA resulting in transcriptional repression (lower left panel). In contrast, the low DNA binding affinity by $\mathrm{P} 75^{\text {gag-v-erbA }}$ results in a changed equilibrium between corepressors and coactivators binding to TR at the AGGACA half site by scavenging of corepressors (lower right panel). $\mathrm{R}$ in circles: receptors; $\mathrm{V}$ in circles, $\mathrm{P} 75^{\text {gag-v-erbA }}$; filled ovals, corepressor

enhancement of TR induced transcription (Figure 4a).

We therefore tested directly if the enhancement of TR induced transcription via the F2A element was an effect of out-titration of corepressors by P75 $5^{\text {gag-v-erbA }}$. For this, cells were transfected with the plasmids encoding TR and c56 along with plasmids encoding the SMRT corepressor and the F2Ax2 element. The result shows that an elevation of the intracellular corepressor levels abolished the enhancement conferred by c56 (Figure 4b). The data are consistent with our hypothesis that $\mathrm{P} 75^{\text {gag-v-erbA }}$ out-titrates corepressors when enhancing TR induced activation. 


\section{Discussion}

\section{P75 ${ }^{\text {gag-v-erbA }}$ in transcriptional control}

We have tested two TREs for their responsiveness to thyroid hormone. These elements originate from the control region of the chicken lysozyme gene and they allow homodimer binding of TR. The mutated TRE, that contains a thymidine to adenosine change at the fourth position in the half sites does not alter the capacity of TR to mediate DNA dependent transcriptional control, but eliminates the capacity of P75 $5^{\text {gag-v-erbA }}$ (and the chimeric protein c56) to bind DNA.

In contrast to steroid hormone receptors, TR possesses a dual function in transcriptional regulation. In absence of hormone it represses and in presence thereof it activates transcription. Transcriptional activation is mediated by a conformational change induced by ligand binding, which abolishes the association with a corepressor in favor of the coactivator. A general view has been that $\mathrm{P} 75^{\text {gag-v-erbA }}$ interferes with TR as a consequence of amino acid changes in the LBD. In the C-terminus, a 9 amino acid deletion removes residues necessary for binding of ligand (Munoz et al., 1988) and coactivators. Therefore, the major mechanisms by which P75 ${ }^{\text {gag-v-erbA }}$ acts in repression involves a constitutive binding of corepressor, leading to repression of its target genes, as well as an inhibition of TR to activate such genes in a ligand dependent manner.

The roles in oncogenesis of other mutations in P75 ${ }^{\text {gag-v-erbA }}$ have however remained obscure. Of the approximately 100 amino acid residues that constitute the N-terminus and the DBD of the TR, only four amino acid residues were altered during the conversion to an oncoprotein. The finding that P75 gag-v-erbA, in contrast to TR, fails to bind TREs containing the AGGACA half site (Schmidt et al., 1998) whereas only the oncoprotein binds efficiently to half sites with a guanosine in the fourth position (Ciana et al., 1998) suggests that the pool of target genes for TR and P75 $5^{\text {gag-v-erbA }}$ may in part differ although a considerable overlap is evident. Our results provide an unexpected consequence of the differences in recognition of target genes: the inability of P75 ${ }^{\text {gag-v-erba }}$ to bind to some of the response elements recognized by $\mathrm{TR}$ enhances their activation by thyroid hormone.

What is the mechanism for this enhancement? It is likely that P75 gag-v-erbA enhances through the AGGACA element for two reasons: the oncoprotein has a low affinity for this element and it fails to bind coactivators.

\section{Role of the amino acids in the N-terminus}

To determine which of the four amino acid residues in the DBD and the N-terminus of P75 ${ }^{\text {gag-verbA }}$ that is responsible for the enhancement on AGGACA half sites, we back mutated them to those found in TR. The two amino acids in the DBD that differ between P75 $5^{\text {gag-v-rba }}$ and TR are located in the conserved P-box of the DBD. The D-box facilitates in several steroid receptors homodimerization in presence of ligand. However, the mutation in the D-box of P75 ${ }^{\text {gag-v-erbA }}$ had little effect on repression and enhancement. The Pbox mutation, on the other hand, was critical since the change from a serine to a glycine in this position altered the transcriptional outcome considerably. This single amino acid of $\mathrm{P} 75^{\text {gag-v-erbA }}$ is responsible for the loss of repression and the concomitant enhancement of transcription via the AGGACA half site motif, as shown by the results with the c56, c1256 and c12456 constructs in Figure $3 \mathrm{a}$. This is of a conceptual interest as this amino acid is located in the region where three amino acid residues are appointed crucial for specific DNA sequence recognition.

The first two amino acid changes in $\mathrm{P} 75^{\text {gag-v-erbA }}$ are located $\mathrm{N}$-terminal to the $\mathrm{DBD}$, in a region that also harbors the activating function AF-1 (Hadzik et al., 1995). However, when these residues were changed to those in TR a less efficient repression through the AGGTCA motifs was observed. This may suggest that the residues specific to $\mathrm{P} 75^{\text {gag-v-erbA }}$ alter the properties of AF-1. Alternatively, as suggested by Wong et al. (1995), these alterations alter the DNA binding properties of P75 ${ }^{\text {gag-v-erbA }}$.

The above suggests that the lack of repression through the AGGACA element is due to a failure to bind this sequence. This also argues against the possibility that coactivator binding to $\mathrm{P} 75^{\text {gag-v-erbA }}$ is responsible for the enhancement of TR dependent activation. The enhancement rather appears to be a result from an imbalance in the ratio of coactivators and corepressors present at the response element. A possible mechanism is that P75 ${ }^{\text {gag-v-erbA }}$ through its loss of DNA binding capacity changes the dynamic equilibrium of corepressors and coactivators that associate to the TR in the transcription factor complex. In this model a soluble P75 ag-v-erbA scavenges corepressors, thus allowing a greater interaction between TR and coactivators on the AGGACA element (Figure 4c). This view is further supported by the fact that the LBD of P75 gag-v-erbA has a similar transcriptional enhancing activity on TR regardless of the response element tested (Figure 4a). The observation that an increase in SMRT expression nullifies the enhancement by c56 (Figure 4b) further supports this notion.

\section{P75 ${ }^{\text {gag-v-erbA }}$ in erythroid cell differentiation}

Normal avian erythroid progenitor cells require T3 for differentiation into erythrocytes (Steinlein et al., 1995). When expressing P75 $5^{\text {gag-v-erbA }}$ in the same cells, the terminal stage of differentiation is no longer reached (Beug et al., 1994). The importance of the DBD and the N-terminus of P75 ${ }^{\text {gag-v-erbA }}$ in the block of differentiation has been suggested to result from P75 ${ }^{\text {gag-v-erbA }}$ sequences of DBD (Bonde et al., 1991). This notion was further supported by our experiments with the c56 and $\mathrm{c} 1-6$ constructs (Bauer et al., 1997). Here, erythroblasts expressing $\mathrm{cl}-6$ and the normal TR allowed the progenitor to mature and differentiate whereas cells that instead expressed c56 were aberrant. It is thus possible that transcriptional enhancement is an important feature of the oncoprotein, a view supported by our finding that c56, but not $\mathrm{c} 1-6$, enhances TR activation via the AGGACA element.

In the search for genes specifically regulated by P75 ${ }^{\text {gag-v-erbA }}$ in erythroblasts, so far only three genes that are repressed have been identified (Disela et al., 1991; Fuerstenberg et al., 1990; Zenke et al., 1988). However, 
a systematic search for genes that are indirectly activated by P75 ${ }^{\text {gag-v-erba }}$ has not been published.

Another feature of $\mathrm{P} 75^{\text {gag-v-erbA }}$ is that it represses transcriptional activation induced by retinoids through the retinoic acid receptors (RARs) (Damm, 1993). Retinoids are of crucial importance in development, and an interesting question in this context is if P75 ${ }^{\text {gag-v-erbA }}$ can enhance RAR activated transcription, and if such an effect is tissue dependent.

\section{Materials and methods}

\section{In vitro mutagenesis}

The DBD and the N-terminal region of a TR variant receptor, V3-pTZ (Pharmacia \& Upjohn), was cloned into the M13 plasmid which was used in site directed in vitro mutagenesis (Amersham). Primers were designed to allow one of the four nucleotides, which diverge between TR and P75 $5^{\text {gag-v-erbA }}$, to be exchanged. Double mutants where both amino acid residue 1 and 2 were exchanged, or where residues 3 and 4 were exchanged, were done in a second step where one of the two mutations were introduced. The resulting mutated DBDs were introduced into the v-erbA background. For cloning reasons the amino acids 5 and 6 of TR were included in the fragment when introduced into the P75 $5^{\text {gag-v-rba }}$. DNA sequencing was done to confirm the mutated nucleotides.

\section{Plasmids}

DNA encoding v-erbA and variant proteins was cloned into a retrovirus vector derivative $(\mathrm{CV})$ of SFCV-LE (Fuerstenberg et al., 1990). TR, v-erbA and the sequences encoding the LBD of v-erbA were subcloned in pSG5 (Sap et al., 1989). pCMX-SMRT expression vector was kindly provided (Chen and Evans, 1995) and the $\beta$-galactosidase reporter plasmid containing a CMV promoter was commercially available (pCH110, Pharmacia \& Upjohn). Response element sequences were synthesized, annealed and cloned into HindIII and BamHI sites of the luciferase vector pTLUC109 (Nordeen, 1988). The F2Tx2 upper strand is: AGCTTATTGACCCCAGCTGA GGTCAAGTTATTGACCCCAGCTGAGGTCAAGTTG and the F2Ax2 upper strand is: AGCTTATTGTC CCCAGCTGAGGACAAGTTATTGTCCCCAGCTGAGGACAAGTTG. The LacZ gene was subcloned into two different vectors, the SFCV-LE retrovirus vector and pSG5.

\section{Cell culture and transfections}

For protein expression studies quail T6 fibroblast cells (Moscovici et al., 1977) were stable transfected with plasmids

\section{References}

Andersson ML, Nordström K, Demczuk S, Harbers M and Vennström B. (1992). Nucl. Acids Res., 20, 4803-4810.

Baniahmad A, Dressel U and Renkawitz R. (1998). Mol. Endocrinol., 12, 504-512.

Baniahmad A, Steiner C, Köhne AC and Renkawitz R. (1990). Cell, 61, 505-514.

Bauer A, Ulrich E, Andersson M, Beug $\mathrm{H}$ and von Lindern M. (1997). Oncogne, 15, 701-715.

Beug H, Mullner EW and Hayman MJ. (1994). Curr. Opin. Cell Biol., 6, 816-824.

Bonde BG, Sharif M and Privalsky M. (1991). J. Virol., 65, 2037-2046. encoding the variant protein using the calcium phosphate technique. $5-10 \mu \mathrm{g}$ of plasmid DNA and $1 \mu \mathrm{g}$ of RCAN helper DNA (Sudol et al., 1986) was transfected into cells in a $50 \mathrm{~mm}$ dish and incubated in $5 \% \mathrm{CO}_{2}$ at $37^{\circ} \mathrm{C}$. Next day the cell medium was exchanged and cells were incubated for another $24-36 \mathrm{~h}$ before they were exposed to media containing the neo resistance drug G418 (Gibco). Seven days after neo selection the virus supernatants were frozen. Viral titers were estimated by infecting QT6 cells, or chicken embryo fibroblasts, with virus supernatants. Viral titers were estimated to approximately $10^{6}$ colonies $/ \mathrm{ml}$.

In transfection assays approximately 500000 QT6 cells were seeded per well in a 24-well plate the day before transfections. The medium was changed the following morning, and $4 \mathrm{~h}$ later supercoiled DNA encoding TR, variant proteins, $\beta$-galactosidase and luciferase were added. Cells were incubated overnight and boosted with $15 \%$ glycerol $15 \mathrm{~h}$ later. Cells were lysed with $150 \mu \mathrm{l}$ cell lysis buffer (BioOrbit) after another $36-40 \mathrm{~h}$ of incubation. Fifty $\mu \mathrm{l}$ was taken to analysis for $\beta$-galactosidase and $50 \mu \mathrm{l}$ for luciferase (Luciferin from Biothema) assay.

\section{Gel mobility analysis}

Cells containing Vaccinia virus produced $\mathrm{P} 75^{\text {gag-v-erbA }}$ or TR (Andersson et al., 1992; Wahlstrom et al., 1996) were lysed and extracts were mixed with 700 fmoles of synthetic ${ }^{32} \mathrm{P}-$ labeled F2Tx 2 or F2Ax2 oligonucleotide together with poly $\mathrm{d}(\mathrm{I}) \mathrm{d}(\mathrm{C})$. The mix was run in a $6 \%$ non-denaturing acrylaminde gel as previously described (Andersson et al., 1992).

\section{Western blot}

Extracts from virus infected QT6 cells containing P75 ${ }^{\text {gag-v-erbA }}$ or variant protein receptors were analysed with $6 \%$ SDSPAGE. Vaccinia virus- and CRA-produced P75 ${ }^{\text {gag-v-erbA }}$ (Sap et al., 1986) were used as controls for the variant receptors (75 kD). A polyclonal antibody recognizing TR and P75 ${ }^{\text {gag-v-erbA }}$ (Sjöberg and Vennström, 1995) was used and the antibody detection was visualized using ECL (Amersham).

\section{Acknowledgements}

We would like to thank $\mathrm{H}$ Beug for discussion, $\mathrm{S}$ Oh for technical help. This project was supported by Cancerfonden, Human Frontiers, the Swedish Society for Medical Research and Goljes Fund.

Casanova J, Helmer E, Selmi-Ruby S, Qi J-S, Au-Fliegner M, Desai-Yajnik V, Koudinova N, Yarm F, Raaka BM and Samuels HH. (1994). Mol. Cell. Biol., 14, 5756- 5765.

Chen H, Smit-McBride, Lewis S, Sharif M and Privalsky ML. (1993). Mol. Cell. Biol., 13, 2366-2376.

Chen JD and Evans RM. (1995). Nature, 377, 454-457.

Chen JD, Umesono K and Evans RM. (1996). Proc. Natl. Acad. Sci. USA, 93, 7567-7571.

Ciana P, Braliou GG, Demay FG, von Lindern M, Barettino D, Beug H and Stunnenberg HG. (1998). EMBO J., 17, $7382-7394$. 
Damm K. (1993). FASEB J., 7, 904-909.

Damm K, Thompson CC and Evans RM. (1989). Nature, 339, 593-597.

Desai-Yajnik V, Hadzik E, Modlinger P, Malhorta S, Gechlik G and Samuels H. (1995). J. Virol., 69, 51035112.

Disela C, Glineur C, Bugge T, Sap J, Stengl G, Dodgson J, Stunnenberg H, Beug H and Zenke M. (1991). Genes Dev., 5, 2033-2047.

Farsetti A, Desvergne B, Hallenbeck P, Robbins J and Nikodem VM. (1992). J. Biol. Chem., 267, 15784-15788.

Fuerstenberg S, Beug H, Introna M, Khazaie K, Munos A, Ness S, Nordström K, Sap J, Stanley I, Zenke M and Vennström B. (1990). J. Virol., 64, 5891-5902.

Glass CK, Franco R, Weinberger C, Albert VR, Evans RM and Rosenfeld MG. (1987). Nature, 329, $738-741$.

Hadzik E, Desai-Yajnik V, Helmer E, Guo S, Wu S, Koudinova N, Casanova J, Raaka BM and Samuels HH. (1995). Mol. Cell. Biol., 15, 4507-4517.

Horlein AJ, Naar AM, Heinzel T, Torchia J, Gloss B, Kurokawa R, Ryan A, Kamei Y, Soderstrom M and Glass CK. (1995). Nature, 377, $397-404$.

Judelson C and Privalsky ML. (1996). J. Biol. Chem., 271, $10800-10805$.

Kurokawa R, Söderström M, Hörlein A, Halachmi S, Brown M, Rosenfeld MG and Glass CK. (1995). Nature, 377 $451-454$.

Lee Y and Mahdavi V. (1993). J. Biol. Chem., 268, $2021-$ 2028.

Li H, Leo C, Schroen DJ and Chen JD. (1997). Mol. Endocrinol., 11, 2025-2037.

Moscovici C, Moscovici MG, Jimenez H, Lai MM, Hayman MJ and Vogt PK. (1977). Cell, 11, 95-103.

Munoz A, Zenke M, Gehring U, Sap J, Beug $\mathrm{H}$ and Vennström B. (1988). EMBO J., 7, 155-159.

Näär AM, Boutin J-M, Lipkin SM, Yu VC, Holloway JM, Glass CK and Rosenfeld MG. (1991). Cell, 65, 12671279.

Nawaz A, Tsai MJ and O’Malley BW. (1995). Proc. Natl. Acad. Sci. USA, 92, $11691-11695$.

Nelson CC, Hendy SC, Faris JS and Romaniuk PJ. (1994). Mol. Endocrinol., 8, 829-840.

Nordeen SK. (1988). Biotechniques, 6, 454-458.

Petty KJ, Desvergne B, Mitsuhashi T and Nikodem VM. (1990). J. Biol. Chem., 265, 7395-7400.

Privalsky ML, Boucher P, Koning A and Judelson C. (1988). Mol. Cell. Biol., 8, 4510-4517.
Rascle A, Ghysdael J and Samarut J. (1994). Oncogene, 9, $2853-2867$.

Sap J, Munoz A, Damm K, Goldberg Y, Ghysdael J, Leutz A, Beug H and Vennström B. (1986). Nature, 324, $635-$ 640.

Sap J, Munoz A, Schmitt J, Stunnenberg H and Vennström B. (1989). Nature, 340, $242-244$

Schmidt S, Baniahmad A, Eggert M, Schneider S and Renkawitz R. (1998). Nucl. Acids Res., 26, 1191 - 1197.

Schroeder C, Gibson L and Beug H. (1992a). Oncogene, 7, $203-216$.

Schroeder C, Gibson L, Zenke M and Beug H. (1992b). Oncogene, 7, 217-227.

Sjöberg M and Vennström B. (1995). Mol. Cell. Biol., 15, $4718-4726$

Smit-McBride Z and Privalsky ML. (1994). Mol. Endocrinol., 8, 819-828.

Steinlein P, Wessely O, Meyer S, Deiner E-M, Hayman MJ and Beug H. (1995). Curr. Biol., 5, 191-204.

Sudol M, Lerner TL and Hanafusa H. (1986). Nucl. Acids Res., 14, $2391-2405$.

Tagami T, Gu WX, Peairs PT, West BL and Jameson JL. (1998). Mol. Endocrinol., 12, $1888-1902$.

Tsika RW, Bahl JJ, Leinwand LA and Morkin E. (1990). Proc. Natl. Acad. Sci. USA, 87, 379-383.

Umesono K, Murakami KK, Thompson CC and Evans RM. (1991). Cell, 65, 1255-1266.

Wahlstrom GM, Harbers M and Vennström B. (1996). Oncogene, 13, 843-852.

Wahlström GM, Sjöberg M, Andersson ML, Nordström K and Vennström B. (1992). Mol. Endocrinol., 6, 10131022 .

Weinberger C, Thompson C, Ong ES, Lebo R, Grvol DJ and Evans RM. (1986). Nature, 324, 641-646.

Wong J, Shi Y and Wolffe AP. (1995). Genes Dev., 9, $2696-$ 2711 .

Wong J, Shi Y and Wolffe AP. (1997). EMBO J., 16, 7130 7145 .

Yen PM, Darling DS, Carter RL, Forgione M, Umeda PK and Chin WW. (1992). J. Biol. Chem., 267, 3565-3568.

Zamir I, Zhang J and Lazar MA. (1997). Genes Dev., 11, $835-846$

Zenke M, Kahn P, Disela C, Vennström B, Leutz A, Keegan K, Hayman MJ, Choi HR, Yew N, Engel JD and Beug H. (1988). Cell, 52, 107-119. 